\title{
无碱条件下由芳香醛肜和磺酰氯制备 O-磺酰基芳香醛肜
}

\author{
邓桂胜*,a,b 莫宏华 ${ }^{a}$ 罗 景 ${ }^{a}$ 汤 力 ${ }^{a}$ \\ ( ${ }^{a}$ 湖南师范大学化学化工学院 长沙 410081) \\ ( ${ }^{b}$ 化学生物学及中药分析教育部重点实验室(湖南师范大学) 长沙 410081)
}

\begin{abstract}
摘要 在氧化银和磑化钾存在下, 磺酰氯与肜室温反应合成一系列 $O$-磺酰基肜. 芳香醛肜和酮肜作为底物, 产率 $56 \% \sim 87 \%$. 不过, 对硝基苯甲醛肜、脂肪醛肜及 $\alpha, \beta$-不饱和醛肜作为该反应底物时, 没有获得目标产物.

关键词 $O$-磺酰基朊; 合成; 肜; 磺酰氯; 氧化银
\end{abstract}

\section{Base-Free Method for Preparation of O-Sulfonyl Aldoximes Starting from Aldoximes and Sulfonyl Chlorides}

\author{
Deng, Guisheng ${ }^{*, a, b} \quad$ Mo, Honghua ${ }^{a} \quad$ Luo, Jing ${ }^{a} \quad$ Tang, Li $^{a}$ \\ ( ${ }^{a}$ College of Chemistry and Chemical Engineering, Hunan Normal University, Changsha 410081) \\ $\left({ }^{b}\right.$ Key Laboratory of Chemical Biology \& Traditional Chinese Medicine Research (Hunan Normal University), \\ Ministry of Education, Changsha 410081)
}

\begin{abstract}
A series of $O$-sulfonyl oxime derivatives have been synthesized by the reaction of oximes with sulfonyl chlorides in the presence of silver oxide and potassium iodide. In the case of aromatic aldoximes and ketoximes, desired products were obtained in $56 \% \sim 87 \%$ yields. But then no desired products were obtained when $p$-nitrobenzaldoxime, aliphatic aldoximes and $\alpha, \beta$-unsaturated aldoximes were employed as substrates in the process.
\end{abstract}

Keywords $O$-sulfonyl oximes; synthesis; oximes; sulfonyl chlorides; silver oxide

$O$-磺酰基肜是重要的有机合成中间体 ${ }^{[14]}$. 它不仅 稳定足以分离纯化，而且能够发生许多重要的转化，例 如, 加成-取代反应 ${ }^{[1,2,5]}$ 、消除成腈 ${ }^{[6,7]} 、 \mathrm{Neber}^{[8,9]}$ 或 Beckmann $^{[10 \sim 12]}$ 重排、亲电胺化 ${ }^{[13,14]} 、 O$-磺酰基肜氮原 子上的亲核取代 ${ }^{[5,15]}$ 、经缩合反应构建碳-碳键 ${ }^{[16]}$ 、 Beckmann 重排-烷基化反应 ${ }^{[17,18]}$ 、热解 ${ }^{[6,7]}$ 、过氧酸氧 化 ${ }^{[19]}$ 等. 在现代光刻技术应用中, $O$-磺酰基肜作为重要 的光致酸发生剂, 已用于半导体芯片的批量生产 ${ }^{[20]}$. 因 此, $O$-磺酰基肜在有机合成中扮演越来越重要的角色.

由于 $O$-磺酰基肜具有广泛的应用潜力, 其合成方 法学研究也备受人们的关注. $O$-磺酰基酮肜最有效的制 备方法是, 在碱存在下, 酮肜与磺酰氯直接反应制

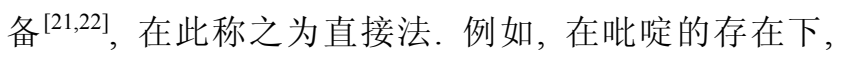
对甲苯磺酰氯与三氟甲基酮肜在 $70{ }^{\circ} \mathrm{C}$ 反应, 制备 $O$-磺 酰基三氟甲基酮肜 ${ }^{[1,23]}$. 然而, 由于 $O$-磺酰基芳香醛肜 对酸、碱、热的敏感性而易分解的缘故, 类似的直接法
制备 $O$-磺酰基芳香醛肜方法极少成功. 例如, 使用强碱 如氨基钠 ${ }^{[24]}$, 或弱碱如三乙胺 ${ }^{[6]}$, 对甲苯磺酰氯与芳香 醛肜的反应均能得到相应 $O$-磺酰基芳香醛肜. 但是, 这 些报道的直接法合成 $O$-磺酰基芳香醛肜存在一些缺陷, 如苛刻的反应条件和繁琐的操作 ${ }^{[24,7]}$ 或低的产率 ${ }^{[6]}$. 产 率低的重要原因是, 在这些反应中, 无论使用强碱还是 弱碱，都容易造成 $O$-磺酰基芳香醛肜脱去磺酸，使其 转换成相应的腈等副产物 ${ }^{[25]}$. 因此, 寻求开发 $O$-磺酰基 芳香醛肜的无碱的合成方法, 是具有一定理论意义和实 用价值的研究课题之一. 最近报道, 在氧化银和碘化钾 存在及温和的反应条件下, 无碱存在时, 聚乙二醇与磺

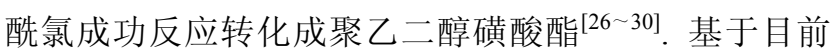
文献制备 $O$-磺酰基芳香醛肜方法存在的不足及发展亚 胺衍生物制备方法学的兴趣 ${ }^{[31]}$, 首次探讨无碱条件下, 磺酰氯与芳香醛肜直接反应制备 $O$-磺酰基芳香醛肜的 方法.

* E-mail: gsdeng@hunnu.edu.cn

Received June 8, 2012; revised June 28, 2012; published online July 11, 2012. 


\section{1 结果与讨论}

在氧化银和碘化钾存在下, $25{ }^{\circ} \mathrm{C}$, 探讨了磺酰氯 2 与一系列肜 1 的反应(表 1). 在此反应过程中, 碘化钾作 为添加剂, 通过卤素交换, 磺酰氯 2 转化成磺酰碘中间 体，从而增强其与弱亲核试剂肜 1 的反应活性，提高反 应速率. 结果表明, 在这种无碱反应体系中, 芳磺酰氯 与多种芳香醛肜反应，得到相应 $O$-磺酰基芳香醛肜 $\mathbf{3}$, 产率 63\% 85\% (表 1, Entries 1 6, 8 10). 与 $\mathrm{NaH}$ 作碱 的文献 ${ }^{[7]}$ 方法仅 $55 \% \sim 60 \%$ 的产率相比, 使用这种无碱 方法，产率明显提高. 在同样条件下，对硝基苯甲醛肜 不与芳磺酰氯反应(表 1, Entry 7). 这是由于对硝基苯甲 醛肟亲核性很差的缘故 ${ }^{[24]}$. 甲磺酰氯与芳香醛肜反应 的产率相对较低(表 1, Entries 11，12). 这是甲磺酰氯比 芳磺酰氯活性较低所致。在上述实例中, 用 TLC 和 NMR 检测，没有发现 $O$-磺酰基芳香醛肜消除形成相应 的腈等副产物, 反应重现性好, 后处理简单. 采用文 献方法 ${ }^{[6,7,24]}$, 能制得相应的 $O$-磺酰基芳香醛肟, 但反应 过程中已有相当部分 $O$-磺酰基芳香醛肜分解成相应的 腈, 反应难以控制, 重现性很差. 大部分 $O$-磺酰基芳香 醛肜相当稳定, 但纯的 $\mathbf{3 f}$ 很不稳定, 空气中室温下放置
过夜，易变成不明的化合物.

此外，这种无碱方法能扩展到 $O$-磺酰基芳香及脂 肪酩肟的制备，产率达 85\%以上(表 1, Entries 13，14). 不过，芳环上具有活性羟基的邻羟基苯乙酮肜，并不反 应产生 $O$-对甲苯磺酰邻羟基苯乙酮肪. 同样地，按文 献方法 ${ }^{[6,7,24]}$ 使用 $\mathrm{NaH}$ 或 $\mathrm{Et}_{3} \mathrm{~N}$ ，也未能制备相应的 $O$-磺 酰基酮肟(表 1，Entry 15)。这表明，芳环上活性着基对 $O$-磺酰肜的形成极为不利.

当使用 $\alpha, \beta$-不饱和醛肜作底物时，没有得到预期的 相应 $O$-磺酰基肟，其产物成分复杂、结构难辨(表 1 , Entries 16，17). 一个合理的解释是，在该反应体系中, 形成的 $O$-磺酰 $\alpha, \beta$-不饱和醛肜不稳定、易分解. 同样条 件下，脂肪族醛肜不与芳磺酰氯反应(表 1, Entries 18, 19).

\section{2 结论}

在这个研究方案中, 首次采用无碱方法, 由芳香醛 肜和磺酰氯直接反应制备 $O$-磺酰基芳香醛肟，产率达 56\% 85\%. 该方法能扩展制备 $O$-磺酰基酮肜，产率达 $85 \%$ 以上. 这种新方法有几个优点：反应条件温和、操 作简单、避免了碱性条件下 $O$-磺酰基芳香醛肜分解成腈

表 1 肜与磺酰氯的无碱反应 ${ }^{a}$

Table 1 Base-free reaction of oximes with sulfonyl chlorides

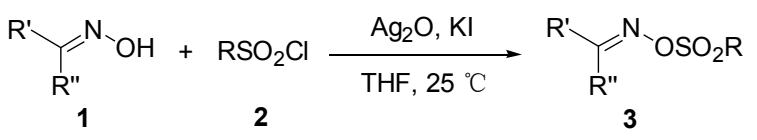

\begin{tabular}{|c|c|c|c|c|c|}
\hline Entry & Product 3 & R'; R" & $\mathrm{R}$ & Reaction time/h & Yield $^{b} \%$ \\
\hline 1 & $3 a$ & $\mathrm{Ph} ; \mathrm{H}$ & Ts & 20 & 70 \\
\hline 2 & $3 \mathbf{b}$ & $p-\mathrm{CH}_{3} \mathrm{C}_{6} \mathrm{H}_{4} ; \mathrm{H}$ & Ts & 20 & 72 \\
\hline 3 & $3 c$ & $p-\mathrm{CH}_{3} \mathrm{OC}_{6} \mathrm{H}_{4} ; \mathrm{H}$ & Ts & 24 & 78 \\
\hline 4 & $3 d$ & $p-\mathrm{ClC}_{6} \mathrm{H}_{4} ; \mathrm{H}$ & Ts & 35 & 79 \\
\hline 5 & $3 e$ & $p-\mathrm{BrC}_{6} \mathrm{H}_{4} ; \mathrm{H}$ & Ts & 35 & 78 \\
\hline 6 & $3 f$ & 2-Furyl; H & Ts & 16 & 63 \\
\hline 7 & $3 g$ & $p-\mathrm{NO}_{2} \mathrm{C}_{6} \mathrm{H}_{4} ; \mathrm{H}$ & Ts & 10 & $\mathrm{NR}^{c}$ \\
\hline 8 & $3 \mathrm{~h}$ & $p-\mathrm{CH}_{3} \mathrm{C}_{6} \mathrm{H}_{4} ; \mathrm{H}$ & $\mathrm{PhSO}_{2}$ & 18 & 85 \\
\hline 9 & $3 \mathbf{i}$ & $p-\mathrm{CH}_{3} \mathrm{OC}_{6} \mathrm{H}_{4} ; \mathrm{H}$ & $\mathrm{PhSO}_{2}$ & 42 & 82 \\
\hline 10 & $3 \mathbf{j}$ & $p-\mathrm{ClC}_{6} \mathrm{H}_{4} ; \mathrm{H}$ & $\mathrm{PhSO}_{2}$ & 18 & 75 \\
\hline 11 & $3 \mathbf{k}$ & $p-\mathrm{CH}_{3} \mathrm{OC}_{6} \mathrm{H}_{4} ; \mathrm{H}$ & $\mathrm{Ms}$ & 42 & 56 \\
\hline 12 & 31 & $p-\mathrm{ClC}_{6} \mathrm{H}_{4} ; \mathrm{H}$ & Ms & 48 & 60 \\
\hline 13 & $3 m$ & $\mathrm{Ph} ; \mathrm{CH}_{3}$ & Ts & 30 & 85 \\
\hline 14 & $3 n$ & $-\left(\mathrm{CH}_{2}\right)_{5}^{-}$ & Ts & 40 & 87 \\
\hline 15 & 30 & $o-\mathrm{HOC}_{6} \mathrm{H}_{4} ; \mathrm{CH}_{3}$ & Ts & 40 & $\mathrm{NR}^{c}$ \\
\hline 16 & $3 p$ & $E-\mathrm{CH}_{3} \mathrm{CH}=\mathrm{CH} ; \mathrm{H}$ & Ts & 10 & $\mathrm{ND}^{d}$ \\
\hline 17 & $3 q$ & $E-\mathrm{PhCH}=\mathrm{CH} ; \mathrm{H}$ & Ts & 10 & $\mathrm{ND}^{d}$ \\
\hline 18 & $3 \mathbf{r}$ & $\left(\mathrm{CH}_{3}\right)_{2} \mathrm{CHCH}_{2} ; \mathrm{H}$ & Ts & 45 & $\mathrm{NR}^{c}$ \\
\hline 19 & $3 s$ & $\mathrm{CH}_{3}\left(\mathrm{CH}_{2}\right)_{3} ; \mathrm{H}$ & Ts & 50 & $\mathrm{NR}^{c}$ \\
\hline
\end{tabular}

$\bar{a}$ The equiv. amounts of oxime $\mathbf{1}, \mathrm{RSO}_{2} \mathrm{Cl} \mathbf{2}, \mathrm{Ag}_{2} \mathrm{O}$ and $\mathrm{KI}$ were $1,1.5,1$ and 1 , respectively; ${ }^{b}$ Isolated yield based on oximes used; ${ }^{c}$ No reaction; ${ }^{d}$ No desired product. 
等副反应，明显提高了反应的产率. 虽然该方法不适用 于 $O$-磺酰基 $\alpha, \beta$-不饱和醛肜及脂肪醛肜的制备, 但对于 $O$-磺酰基芳香醛肜的制备, 它具有重要的意义.

\section{3 实验部分}

\section{1 仪器与试剂}

所有溶剂和试剂未处理, 直接使用. 熔点通过 YRT-3 仪测量, 但未校正. 红外光谱(IR)通过 NICOLET NEXUS TT-IR 仪记录. ${ }^{1} \mathrm{H}$ NMR 和 ${ }^{13} \mathrm{C}$ NMR 使用 Bruker Avance-500 光谱仪记录, TMS 作为内标, $\mathrm{CDCl}_{3}$ 作溶剂. MS(FAB)和 HRMS 分别使用 Waters ZQ2000 和 Bruker APEX IV 检测. 使用默克公司 25 薄层铝板材(硅)胶 60 GF 254, 0.25 毫米)进行薄层色谱法监测反应. 通过硅胶 ZCX- $\alpha(300 \sim 400$ 目)制备柱色谱分离纯化获得元素分 析用高纯品. 所有已知化合物的光谱和物理数据与标准 的样本对照.

\section{2 无碱条件下肜与磺酰氯反应制备 O-磺酰基肟的一 般过程}

以 $O$-对甲基苯磺酰基苯甲醛肜 3a 的制备为例. 将 $\mathrm{Ag}_{2} \mathrm{O}(5 \mathrm{mmol}, 1.200 \mathrm{~g})$, KI (5 mmol, $0.850 \mathrm{~g}$ )和对甲苯 磺酰氯 $(7.5 \mathrm{mmol}, 1.425 \mathrm{~g}$ )加到快速摚拌的苯甲醛肜 $(5$ $\mathrm{mmol}, 0.605 \mathrm{~g})$ 的 $50 \mathrm{~mL}$ 四氢呋喃溶液中. 反应混合物在 $25{ }^{\circ} \mathrm{C}$ 摚拌 $20 \mathrm{~h}$. 过滤、残留物用二氯甲烷 $(15 \mathrm{~mL} \times 2)$ 洗涤. 合并滤液, 减压浓缩得棕色油状粗产品. 加入石 油醚洗涤, 析出固体, 抽滤收集固体. 然后用乙醚/石油 醚 $(V: V=1: 1)$ 重结晶, 得到纯产品 $\mathbf{3 a}(0.910 \mathrm{~g})$, 产率 $70 \%$. 白色固体, m.p. $84 \sim 85{ }^{\circ} \mathrm{C}$ (lit. ${ }^{[24]} 85 \sim 86{ }^{\circ} \mathrm{C}$ ). ${ }^{1} \mathrm{H}$ NMR $\left(\mathrm{CDCl}_{3}, 500 \mathrm{MHz}\right) \delta: 8.23(\mathrm{~s}, 1 \mathrm{H}), 7.26 \sim 7.98(\mathrm{~m}$, $\mathrm{H}), 2.44(\mathrm{~s}, 3 \mathrm{H})$; IR (KBr) v: $1675(\mathrm{~m}, \mathrm{C}=\mathrm{N}), 1175(\mathrm{~s}$, $\left.\mathrm{SO}_{3}\right) \mathrm{cm}^{-1}$.

3b: 产率 $72 \%$. 无色固体, m.p. $112 \sim 113{ }^{\circ} \mathrm{C}$ (lit. ${ }^{[24]}$ $\left.100 \sim 102{ }^{\circ} \mathrm{C}\right) ;{ }^{1} \mathrm{H}$ NMR $\left(\mathrm{CDCl}_{3}, 500 \mathrm{MHz}\right) \delta: 8.19$ (s, $1 \mathrm{H}), 7.92(\mathrm{~d}, J=8.0 \mathrm{~Hz}, 2 \mathrm{H}), 7.46$ (d, $J=8.0 \mathrm{~Hz}, 2 \mathrm{H}), 7.35$ (d, $J=8.0 \mathrm{~Hz}, 2 \mathrm{H}), 7.19$ (d, $J=8.0 \mathrm{~Hz}, 2 \mathrm{H}), 2.44$ (s, 3H), $2.37(\mathrm{~s}, 3 \mathrm{H}) ; \mathrm{IR}(\mathrm{KBr}) v: 1650(\mathrm{~m}, \mathrm{C}=\mathrm{N}), 1178\left(\mathrm{~s}, \mathrm{SO}_{3}\right)$ $\mathrm{cm}^{-1}$.

3c: 产率 78\%. 无色固体, m.p. $109 \sim 110{ }^{\circ} \mathrm{C}$ (lit. ${ }^{[24]}$ 99 100 $\left.{ }^{\circ} \mathrm{C}\right) ;{ }^{1} \mathrm{H} \mathrm{NMR}\left(\mathrm{CDCl}_{3}, 500 \mathrm{MHz}\right) \delta: 8.17(\mathrm{~s}, 1 \mathrm{H})$, $7.92(\mathrm{~d}, J=8.0 \mathrm{~Hz}, 2 \mathrm{H}), 7.52(\mathrm{~d}, J=8.0 \mathrm{~Hz}, 2 \mathrm{H}), 7.35(\mathrm{~d}$, $J=8.0 \mathrm{~Hz}, 2 \mathrm{H}), 6.89$ (d, $J=8.0 \mathrm{~Hz}, 2 \mathrm{H}), 3.83$ (s, 3H), 2.17 (s, 3H); IR (KBr) v: $1661(\mathrm{~m}, \mathrm{C}=\mathrm{N}), 1176\left(\mathrm{~s}, \mathrm{SO}_{3}\right) \mathrm{cm}^{-1}$.

3d: 产率 79\%. 无色固体, m.p. $109 \sim 110{ }^{\circ} \mathrm{C}$ (lit. ${ }^{[7]}$ $\left.109 \sim 110{ }^{\circ} \mathrm{C}\right) ;{ }^{1} \mathrm{H}$ NMR $\left(\mathrm{CDCl}_{3}, 500 \mathrm{MHz}\right) \delta: 8.23$ (s, $1 \mathrm{H}), 7.93(\mathrm{~d}, J=8.0 \mathrm{~Hz}, 2 \mathrm{H}), 7.57(\mathrm{~d}, J=8.0 \mathrm{~Hz}, 2 \mathrm{H})$, 7.35 7.48 (m, 4H), 2.45 (s, 3H); IR (KBr) v: 1660 (m,
$\mathrm{C}=\mathrm{N}), 1175\left(\mathrm{~s}, \mathrm{SO}_{3}\right) \mathrm{cm}^{-1}$.

3e: 产率 78\%. 无色固体, m.p. $116 \sim 117{ }^{\circ} \mathrm{C}$ (lit. ${ }^{[24]}$ 106 107 $\left.{ }^{\circ} \mathrm{C}\right)$; ${ }^{1} \mathrm{H}$ NMR $\left(\mathrm{CDCl}_{3}, 500 \mathrm{MHz}\right) \delta: 8.19$ (s, $1 \mathrm{H}), 7.91(\mathrm{~d}, J=8.0 \mathrm{~Hz}, 2 \mathrm{H}), 7.53(\mathrm{~d}, J=8.0 \mathrm{~Hz}, 2 \mathrm{H}), 7.44$ (d, $J=8.0 \mathrm{~Hz}, 2 \mathrm{H}), 7.36$ (d, $J=8.0 \mathrm{~Hz}, 2 \mathrm{H}), 2.45$ (s, 3H); IR (KBr) $v: 1659(\mathrm{~m}, \mathrm{C}=\mathrm{N}), 1175\left(\mathrm{~s}, \mathrm{SO}_{3}\right) \mathrm{cm}^{-1}$.

3f: 产率 $63 \%$. 无色固体, 熔融分解; ${ }^{1} \mathrm{H}$ NMR $\left(\mathrm{CDCl}_{3}, 500 \mathrm{MHz}\right) \delta: 8.10(\mathrm{~s}, 1 \mathrm{H}), 7.89(\mathrm{~d}, J=8.3 \mathrm{~Hz}$, 2H), $7.50(\mathrm{~d}, J=1.4 \mathrm{~Hz}, 1 \mathrm{H}), 7.33(\mathrm{~d}, J=8.1 \mathrm{~Hz}, 2 \mathrm{H}), 6.84$ (d, $J=3.5 \mathrm{~Hz}, 1 \mathrm{H}), 6.46$ (dd, $J=3.5,1.8 \mathrm{~Hz}, 1 \mathrm{H}), 2.39$ (s, $3 \mathrm{H}) ;{ }^{13} \mathrm{C} \mathrm{NMR}\left(\mathrm{CDCl}_{3}, 126.8 \mathrm{MHz}\right) \delta: 146.40,146.28$, $145.27,144.22,132.22,129.69,128.98,117.28,112.19$, 21.71; IR (KBr) v: $1661(\mathrm{~m}, \mathrm{C}=\mathrm{N}), 1178\left(\mathrm{~s}, \mathrm{SO}_{3}\right) \mathrm{cm}^{-1}$. HRMS calcd for $\left[\mathrm{C}_{12} \mathrm{H}_{11} \mathrm{NO}_{4} \mathrm{~S}+\mathrm{H}\right]$ : 266.0487, found 266.0489. Anal. calcd for $\mathrm{C}_{12} \mathrm{H}_{11} \mathrm{NO}_{4} \mathrm{~S}$ : C 54.33, H 4.18, N 5.28. found C 54.21, H 4.36, N 5.22.

3h: 产率 $85 \%$. 无色固体, m.p. 87 $89{ }^{\circ} \mathrm{C} ;{ }^{1} \mathrm{H}$ NMR $\left(\mathrm{CDCl}_{3}, 500 \mathrm{MHz}\right) \delta: 8.20(\mathrm{~s}, 1 \mathrm{H}), 8.04(\mathrm{~d}, J=8.0 \mathrm{~Hz}$, $2 \mathrm{H}), 7.65(\mathrm{t}, J=7.5 \mathrm{~Hz}, 1 \mathrm{H}), 7.56$ (t, $J=7.8 \mathrm{~Hz}, 2 \mathrm{H}), 7.45$ (d, $J=8.0 \mathrm{~Hz}, 2 \mathrm{H}), 7.18(\mathrm{~d}, J=8.0 \mathrm{~Hz}, 2 \mathrm{H}), 2.35$ (s, 3H); ${ }^{13} \mathrm{C} \mathrm{NMR}\left(\mathrm{CDCl}_{3}, 126.8 \mathrm{MHz}\right) \delta: 157.16,142.90,135.59$, 134.15, 129.77, 129.11, 128.97, 128.39, 126.37, 21.71; IR (KBr) $v: 1658(\mathrm{~m}, \mathrm{C}=\mathrm{N}), 1172\left(\mathrm{~s}, \mathrm{SO}_{3}\right) \mathrm{cm}^{-1}$. HRMS calcd for $\left[\mathrm{C}_{14} \mathrm{H}_{13} \mathrm{NO}_{3} \mathrm{~S}+\mathrm{H}\right]$ : 276.0694 , found 276.0689. Anal. calcd for $\mathrm{C}_{14} \mathrm{H}_{13} \mathrm{NO}_{3} \mathrm{~S}$ : C 61.07, H 4.76, N 5.09. found $\mathrm{C} 61.14, \mathrm{H} 4.86, \mathrm{~N} 5.03$.

3i: 产率 $82 \%$. 无色固体, m.p. $116 \sim 117{ }^{\circ} \mathrm{C}$ (lit. ${ }^{[24]}$ $\left.99 \sim 100{ }^{\circ} \mathrm{C}\right) ;{ }^{1} \mathrm{H}$ NMR $\left(\mathrm{CDCl}_{3}, 500 \mathrm{MHz}\right) \delta: 3.83(\mathrm{~s}, 3 \mathrm{H})$, $6.88(\mathrm{~d}, J=7.5 \mathrm{~Hz}, 2 \mathrm{H}), 7.50 \sim 8.03(\mathrm{~m}, 5 \mathrm{H}), 8.05(\mathrm{~d}, J=$ $7.0 \mathrm{~Hz}, 2 \mathrm{H}), 8.18(\mathrm{~s}, 1 \mathrm{H})$; IR (KBr) $v: 1660(\mathrm{~m}, \mathrm{C}=\mathrm{N})$, $1179\left(\mathrm{~s}, \mathrm{SO}_{3}\right) \mathrm{cm}^{-1}$.

3j: 产率 75\%. 无色固体, m.p. $115 \sim 117{ }^{\circ} \mathrm{C} ;{ }^{1} \mathrm{H}$ $\operatorname{NMR}\left(\mathrm{CDCl}_{3}, 500 \mathrm{MHz}\right) \delta: 8.22(\mathrm{~s}, 1 \mathrm{H}), 8.09 \sim 8.01(\mathrm{~m}$, 2H), $7.68(\mathrm{t}, J=7.5 \mathrm{~Hz}, 1 \mathrm{H}), 7.58(\mathrm{t}, J=7.8 \mathrm{~Hz}, 2 \mathrm{H}), 7.52$ (d, $J=8.5 \mathrm{~Hz}, 2 \mathrm{H}), 7.37(\mathrm{~d}, J=8.5 \mathrm{~Hz}, 2 \mathrm{H}) ;{ }^{13} \mathrm{C} \mathrm{NMR}$ $\left(\mathrm{CDCl}_{3}, 126.8 \mathrm{MHz}\right) \delta: 155.87,138.32,135.33,134.24$, $129.50,129.37,129.11,128.93,127.65$; IR (KBr) $v: 1660$ $(\mathrm{m}, \mathrm{C}=\mathrm{N}), 1181 \quad\left(\mathrm{~s}, \mathrm{SO}_{3}\right) \mathrm{cm}^{-1}$. HRMS calcd for $\left[\mathrm{C}_{13} \mathrm{H}_{10} \mathrm{ClNO}_{3} \mathrm{~S}+\mathrm{H}\right]:$ : 296.0148, found 296.0151. Anal. calcd for $\mathrm{C}_{13} \mathrm{H}_{10} \mathrm{ClNO}_{3} \mathrm{~S}: \mathrm{C} 52.80, \mathrm{H} 3.41, \mathrm{~N} 4.74$. found $\mathrm{C}$ 52.70, H 3.49, N 4.80 .

3k: 产率 56\%. 无色固体, m.p. 99 101 ${ }^{\circ} \mathrm{C}$ (lit. ${ }^{[7]}$ 99 101 $\left.{ }^{\circ} \mathrm{C}\right) ;{ }^{1} \mathrm{H}$ NMR $\left(\mathrm{CDCl}_{3}, 500 \mathrm{MHz}\right) \delta: 8.12(\mathrm{~s}, 1 \mathrm{H})$, $6.89(\mathrm{~d}, J=8.0 \mathrm{~Hz}, 2 \mathrm{H}), 8.10(\mathrm{~d}, J=8.0 \mathrm{~Hz}, 2 \mathrm{H}), 3.85(\mathrm{~s}$, $3 \mathrm{H}), 2.90(\mathrm{~s}, 3 \mathrm{H})$; IR (KBr) v: $1651(\mathrm{~m}, \mathrm{C}=\mathrm{N}), 1176(\mathrm{~s}$, 
$\left.\mathrm{SO}_{3}\right) \mathrm{cm}^{-1}$.

31: 产率 $60 \%$. 无色固体, m.p. 84 85 C (lit. ${ }^{[31]} 83 \sim$

$\left.85{ }^{\circ} \mathrm{C}\right) ;{ }^{1} \mathrm{H}$ NMR $\left(\mathrm{CDCl}_{3}, 500 \mathrm{MHz}\right) \delta: 8.33(\mathrm{~s}, 1 \mathrm{H}), 7.43$ $(\mathrm{d}, J=8.5 \mathrm{~Hz}, 2 \mathrm{H}), 7.27$ (d, $J=8.5 \mathrm{~Hz}, 2 \mathrm{H}), 3.24$ (s, 3H); IR $(\mathrm{KBr}) v: 1657(\mathrm{~m}, \mathrm{C}=\mathrm{N}), 1173\left(\mathrm{~s}, \mathrm{SO}_{3}\right) \mathrm{cm}^{-1}$.

3m: 产率 85\%. 无色固体, m.p. 59 61 ${ }^{\circ} \mathrm{C}$ (lit. ${ }^{[32]}$ $\left.58 \sim 60{ }^{\circ} \mathrm{C}\right) ;{ }^{1} \mathrm{H} \mathrm{NMR}\left(\mathrm{CDCl}_{3}, 500 \mathrm{MHz}\right) \delta: 7.84(\mathrm{~d}, J=$ $8.2 \mathrm{~Hz}, 2 \mathrm{H}), 7.40 \sim 7.31(\mathrm{~m}, 7 \mathrm{H}), 2.42(\mathrm{~s}, 3 \mathrm{H}), 2.21$ (s, $3 \mathrm{H})$; IR (KBr) v: $1640(\mathrm{~m}, \mathrm{C}=\mathrm{N}), 1180\left(\mathrm{~s}, \mathrm{SO}_{3}\right) \mathrm{cm}^{-1}$.

3n: 产率 $87 \%$. 无色固体, m.p. 58 59 ${ }^{\circ} \mathrm{C}$ (lit. ${ }^{[32]}$ $\left.56.9 \sim 58{ }^{\circ} \mathrm{C}\right) ;{ }^{1} \mathrm{H} \mathrm{NMR}\left(\mathrm{CDCl}_{3}, 500 \mathrm{MHz}\right) \delta: 7.90(\mathrm{~d}, J=$ $8.5 \mathrm{~Hz}, 2 \mathrm{H}), 7.12$ (d, $J=8.5 \mathrm{~Hz}, 2 \mathrm{H}), 2.38$ (s, $3 \mathrm{H}), 1.38 \sim$ $1.93(\mathrm{~m}, 6 \mathrm{H})$; IR (KBr) v: $1648(\mathrm{~m}, \mathrm{C}=\mathrm{N}), 1170\left(\mathrm{~s}, \mathrm{SO}_{3}\right)$ $\mathrm{cm}^{-1}$.

\section{References}

[1] Mikhailyuk, A. N.; Makhova, N. N.; Bova, A. E.; Khmel'nitskii, L. I.; Novikov, S. S. Izv. Akad. Nauk SSSR, Ser. Khim. 1978, 1566 [Bull. Acad. Sci. USSR, Div. Chem. Sci. 1978, 27, 1367 (Engl. Transl.)].

[2] Yamamoto, Y.; Mizuno, H.; Tsuritani, T.; Mase, T. J. Org. Chem. 2009, 74, 1394.

[3] Rad, M. N. S.; Khalafi-Nezhad, A.; Behrouz, S.; Amini, Z.; Behrouz, M. Synth. Commun. 2010, 40, 2429.

[4] Oskooie, H. A.; Heravi, M. M.; Jaddi, Z.; Ghassemzadeh, M. Phosphorus, Sulfur Silicon Relat. Elem. 2005, 180, 1993.

[5] Kim, S.; Kamaldin, N. A. B.; Kang, S.; Kim, S. Chem. Commun. 2010, 46, 7822.

[6] AI-Awadi, N. A.; Elnagdi, M. H.; Kaul, K. Tetrahedron 1998, 54, 4633.

[7] AI-Awadi, N. A.; Elnagdi, M. H.; Kaul, K.; Ilingovan, S.; El-Dusouqui, O. M. E. J. Phys. Org. Chem. 1999, 12, 654.

[8] O'Brine, C. Chem. Rev. 1964, 64, 81.

[9] Ooi, T.; Takahashi, M.; Doda, K.; Maruoka, K. J. Am. Chem. Soc. 2002, 124, 7640 .

[10] Tamura, Y.; Fujiwara, H.; Sumoto, K.; Ikeda, M.; Kita, Y. Synthesis 1973, 215 .
[11] Popp, F. D.; McEwen, W. E. Chem. Rev. 1958, 58, 321.

[12] Hünig, S.; Gräßmann, W.; Meuer, V.; Lücke, E. Chem. Ber. 1967, 100,3024

[13] Hassner, A.; Patchornik, G.; Pradhan, T. K.; Kumareswaran, R. J. Org. Chem. 2007, 72, 658.

[14] Kitamura, M.; Chiba, S.; Narasaka, K. Bull. Chem. Soc. Jpn. 2003, $76,1063$.

[15] Shimada, K.; Moro-oka, A.; Maruyama, A.; Fujisawa, H.; Saito, T.; Kawamura, R.; Kogawa, H.; Sakuraba, M.; Takata, Y.; Aoyagi, S.; Takikawa Y.; Kabuto, C. Bull. Chem. Soc. Jpn. 2007, 80, 567.

[16] Matsumura, Y.; Fujiwara, J.; Maruoka, K.; Yamamoto, H. J. Am. Chem. Soc. 1983, 105, 6312.

[17] Hattori, K.; Maruoka, K.; Yamamoto, H. Tetrahedron Lett. 1982, $23,3395$.

[18] Hattori, K.; Matsumura, Y.; Miyazaki, T.; Yamamoto, H. J. Am. Chem. Soc. 1981, 103, 7368

[19] Davis, F. A.; Nadir, U. K.; Kluger, E. W. J. Chem. Soc., Chem. Commun. 1977, 25.

[20] Yamamoto, H.; Asakura, T.; Nishimae, Y.; Matsumoto, A.; Tanabe, J.; Birbaum, J.-L.; Murer, P.; Hintermann, T.; Ohwa, M. J. Photopolym. Sci. Technol. 2007, 20, 637 .

[21] Doney, J.; Heathcock, C. H. Org. Synth. Coll. 1993, 8, 568.

[22] Costa, A.; Mestres, R.; Riego, J. M. Synth. Commun. 1982, 12, 1003.

[23] Smith, D. P.; Anderson, J.; Plante, J.; Ashcroft, A. E.; Radford, S. E.; Wilson, A. J.; Parker, M. J. Chem. Commun. 2008, 44, 5728.

[24] Crawford, R. J.; Woo, C. Can. J. Chem. 1965, 43, 1534.

[25] Campbell Jr., J. B.; Davenport, T. V. Synth. Commun. 1989, 19, 2255.

[26] Ghosh, S.; Ramakrishnan, S. Angew. Chem. 2004, 116, 3326.

[27] Bouzide, A.; Sauvé, G. Org. Lett. 2002, 4, 2329.

[28] Amela-Cortes, M.; Roullier, V.; Wolpert, C.; Neubauer, S.; Kessler, H.; Bedel, O.; Mignani, S.; Marchi-Artzner, V. Chem. Commun. 2011, 47, 1246.

[29] Zhang T.; Wu, Y.; Pan, X.; Zheng, Z.; Ding, X.; Peng, Y. Eur. Polym. J. 2009, 45, 1625.

[30] Deng, G. S.; Zou, J. Y.; Sun, T. F. Chin. Chem. Lett. 2011, $22,511$.

[31] Ke, S.; Zhang, Z.; Yang, Z.; Wang, K.; Liang, Y.; Zhang, Y.; Long, T.; Jiang, A. CN 102030680, 2011 [Chem. Abstr. 2011, 154, 486054].

[32] Heldt, W. Z. J. Am. Chem. Soc. 1958, 80, 5880 . 